Article

\title{
Effect of Hydrophobic Pollution on Response of Thermo-Sensitive Hydrogel
}

\author{
Hideo Tajima ${ }^{*}$, Fumiaki Sato ${ }^{\dagger}$ and Kazuaki Yamagiwa ${ }^{\dagger}$ \\ Graduate School of Science \& Technology, Niigata University, 2-8050 Ikarashi, Nishi-ku, \\ Niigata 950-2181, Japan; E-Mails: satou.fumiaki@ma.mrc.co.jp (F.S.); \\ yamagiwa@eng.niigata-u.ac.jp (K.Y.)
}

$\dagger$ These authors contributed equally to this work.

* Author to whom correspondence should be addressed; E-Mail: h_tajima@eng.niigata-u.ac.jp; Tel.: +81-25-262-7277; Fax: +81-25-262-6781.

Received: 1 July 2013; in revised form: 10 September 2013 / Accepted: 16 September 2013 /

Published: 30 September 2013

\begin{abstract}
Hydrogels are widely studied for chemical sensors. However, they are known to adsorb organic compound and metal ions. The adsorption abilities of hydrogels against organic compounds and metal ions will negatively affect the performance of a hydrogel based chemical sensor. To clarify the effect of hydrophobic pollution on swelling behavior of temperature-sensitive gel, the temperature-responses of spherical N,N-diethylacrylamide (DEAA) gel in phenol solution were evaluated using the collective polymer diffusion constant. Phenol was selected as a model hydrophobic pollution. The equilibrium radius of DEAA gel changed discontinuously at about $874 \mathrm{~g} / \mathrm{m}^{3}$ phenol solution, and the collective polymer diffusion constant decreased sharply between 874 and $916 \mathrm{~g} / \mathrm{m}^{3}$, suggesting a "critical slowing down". The phenol concentration difference $E_{C}$ was successfully used to correlate phenol concentration with the collective polymer diffusion constant. The correlation will be useful as an estimation of hydrogel response reduction associated with hydrophobic pollution.
\end{abstract}

Keywords: thermo-sensitive gels; hydrophobic pollution; swelling behavior; adsorption; $\mathrm{N}, \mathrm{N}$-diethylacrylamide; phenol 


\section{Introduction}

Hydrogels are widely studied for chemical sensors [1-7] because of their large, reversible changes in volume and other properties in response to small changes in temperature. Shin et al. prepared an inverse opal hydrogel $\mathrm{pH}$ sensor. This $\mathrm{pH}$ sensor showed rapid response time [1]. Lee et al. detected dissolved oxygen and $\mathrm{pH}$ with a hydrogel microarray sensor [2]. Selective detection of heavy metal ions was investigated with microcapsules consisting of a hydrogel and crown ether derivative by Pi et al. [3]. Hydrogel based sensors are also applied to a detection of gaseous material such as $\mathrm{CO}_{2}$ [4], $\mathrm{Cl}_{2}$ [5], $\mathrm{NO}$ [6], and VOCs [7]. On the other hand, hydrogels have a good performance for recyclable adsorbents [8-12] and biomedical applications (including drug delivery) [13-15]. They are not only organic compounds but also absorb metal ions. The adsorption of metal ions by hydrogels has recently attracted increasing attention over the past several years $[10,11,16]$. These adsorption abilities of hydrogels against organic compounds and metal ions will negatively affect a performance of hydrogel based chemical sensor.

In applications of thermo-response hydrogels as a chemical sensor, the important gel characteristics are not only selectivity but also the kinetics of stimulus-response. It is well known the hydrogel kinetics depends on the hydrogel particle radius [17] and shape [18]. In addition, the swelling and shrinking rates of hydrogel change depending on the initial and final temperatures in the temperature swing process [19]. However, limited information is available on the effect of volume change and adsorption process on hydrogel response kinetics. Hydrogels as chemical sensors must operate in a solution included in the target material. However, the swelling ratios of hydrogels vary with changing solute concentrations [20].

Our previous study [21] has already elucidated the dominant factors governing the temperature-response rate of spherical poly(N,N-diethylacrylamide) (DEAA) in pure water. The aim of this study was to elucidate the effects of hydrophobic pollution on the temperature response of hydrogel. This study used polyDEAA gel as a hydrogel and phenol as a model hydrophobic pollution. Phenol is representative of possible contamination in biological and chemical substances. At the same time, phenol gives very specific interaction with polymeric gels. Spherical polyDEAA particles with radii of less than $1 \mathrm{~mm}$ were prepared by the method developed in our previous work [22] and used to measure the temperature response rate because small spherical gel particles have isotropic and rapid volume changes [18,21].

\section{Experimental Section}

\subsection{Temperature-Response of a Single Spherical DEAA Gel Particle in Dilute Phenol Solution}

The temperature-sensitive monomer N,N-diethylacrylamide (DEAA, Kojin Co. Ltd., Japan) was used to prepare the hydrogel materials. DEAA gels were prepared from a liquid-liquid dispersion using static mixing technology, and photo-crosslinked by UV irradiation. The preparation method for polyDEAA particles has been reported in detail previously [22]. Some sample gel particles (single spherical gels) were collected from the resultant polyDEAA gels after the gels were swollen in deionized water at $293 \mathrm{~K}$. 
The calculation theory and method are the same as reported previously [21]. The details are given in the reference and described briefly here.

Because the lower critical solution temperature (LCST) of polyDEAA in water is about $306 \mathrm{~K}$ [23], the initial and final temperatures for swelling were fixed at $313 \mathrm{~K}$ and $293 \mathrm{~K}$, respectively, to include the LCST for adsorption operation. The apparatus consisted of the measuring cell, two thermostatted water baths for initial and final temperature setting, a video microscope, and a PC for video capture. The temperatures of the water baths were controlled to within $\pm 0.1 \mathrm{~K}$. The measuring cell contained a polyDEAA gel particle (the circularity of the gel particle was 1.107) and solution (deionized water or phenol solution). The time course of the volume change of the polyDEAA gel particle was observed with the video microscope and recorded as a digital movie. The swelling experiments described above were carried out at least twice under the same conditions. Photographic images of the sample particle were taken from the digital movie, and the digitalized images were analyzed with a PC. The Heywood diameter was used to evaluate the representative length of the polyDEAA gel particle.

The swelling behavior was analyzed by the following equation based on the Tanaka-Fillmore theory [17]:

$$
\begin{gathered}
\frac{a_{e}-a(t)}{a_{e}-a_{0}}=\frac{6}{\pi^{2}} \sum_{n=1}^{\infty} \frac{1}{n^{2}} \exp \left(-\frac{n^{2} t}{\tau}\right) \\
k_{v}=\frac{1}{\tau}=\frac{\pi^{2} D}{a_{e}^{2}}
\end{gathered}
$$

where $a_{\mathrm{e}}, a_{0}$ and $a(\mathrm{t})$ are the hydrogel characteristic length at equilibrium, initially and at time $t$, and $\tau$ is the relaxation time of the polymer network. When $\tau$ is smaller than time $t$ (that is $t / \tau>1$ ), we can assume $n=1$. It has been already confirmed for the DEAA gel used in this study that linear behavior was within $t / \tau>0.25$ in water [21]. Then we assumed $n=1$, and calculated $k_{v}$ of polyDEAA gel by fitting the experimental data to Equation (1). The collective polymer diffusion constant $D$ could be obtained from the $k_{v}$ value according to Equation (2). To validate the swelling rate of the polyDEAA gel prepared in this study, its response rate was measured in pure water. The $k_{v}$ values are in agreement with the literature data [21]. The collective polymer diffusion constant in pure water, $D_{0}$, was evaluated as $3.95 \times 10^{-11} \mathrm{~m}^{2} \cdot \mathrm{s}^{-1}$ for swelling. This study used polyDEAA gel particles with radii of $0.35-0.50 \mathrm{~mm}$ (as measured in pure water at $293 \mathrm{~K}$ ).

\subsection{Phenol Adsorption Isotherm}

Phenol (99\%, Wako Pure Chemical Industries, Ltd., Osaka, Japan) was used as a model hydrophobic pollution. Phenol adsorption isotherms were measured at $313 \mathrm{~K}$ and $293 \mathrm{~K}$. Gel particles $0.25 \mathrm{~g}$ in dry weight were packed into a filter made of polyterephthalate, and then the packed gels were swollen in deionized water at a given temperature before adsorption isotherm measurement. Before use, we confirmed that the polyterephthalate filter did not adsorb phenol. The packed gels were placed in $50 \mathrm{~cm}^{3}$ of aqueous phenol solution in a vial at the given temperature and allowed to sit for at least a day, with occasional shaking, to reach equilibrium. The phenol concentration in the bulk solution was determined by measuring the UV-absorbance at $270 \mathrm{~nm}$ with a spectrophotometer. The phenol adsorption amount in equilibrium $q_{\text {phenol }}$ was calculated from the material balance of the bulk phenol 
solution. In this study, the volume change of aqueous phenol solution by the water content in swollen hydrogel was ignored because the volume change was estimated to be less than $1 \%$. Although there has been an example reported of the Hill equation approximation for the adsorption isotherm of phenol on a hydrogel [24], the phenol adsorption isotherm was approximately expressed by a Freundlich type equation from an engineering viewpoint:

$$
q_{\text {phenol }}=K \cdot C_{\text {pheno، }}^{1 / N}
$$

where $K$ and $N$ are experimentally determined parameters.

Adsorption isotherm data are shown in Figure 1. At the phenol concentrations used in this study, the phenol adsorption isotherm could be approximately expressed by a Freundlich type equation. The parameter values were estimated as $K=3.34 \times 10^{-4}, 1 / N=1.74$ at $293 \mathrm{~K}$ and $K=1.57 \times 10^{-2}$, $1 / N=1.27$ at $313 \mathrm{~K}$. Because there is one polyDEAA gel particle in an excess amount of phenol solution in this study, the temperature swing operation at a constant bulk phenol concentration will be as indicated by arrow (a) in Figure 1. The arrow (b) in Figure 1 shows the temperature swing operation when the amount of phenol adsorbed is kept constant. The given Freundlich type equation was used to calculate suitable phenol concentrations for swelling of DEAA gel under a constant amount of adsorbed phenol.

Figure 1. Adsorption isotherm data for N,N-diethylacrylamide (DEAA) gel and phenol solution system. Solid lines are fits to a Freundlich type equation.

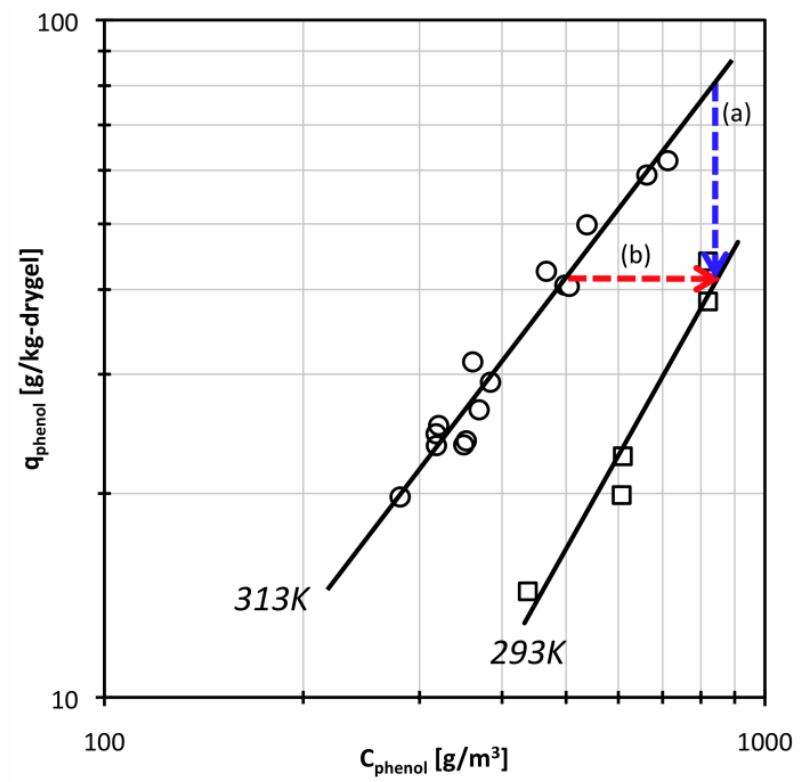

\subsection{Swelling Behavior of DEAA Gel at Fixed amount of Adsorption Phenol}

To evaluate the effect of adsorbed phenol amount on swelling behavior of hydrogel, the adsorbed phenol amount needs to be fixed during the swelling process. This can be done by measuring the swelling behavior with simultaneous changing the bulk phenol concentration and temperature. The bulk phenol concentration, which $q_{\text {phenol }}$ at $293 \mathrm{~K}$ is equal to that at $313 \mathrm{~K}$, was calculated from Equation (3). A specially designed measuring cell was made to easily change the phenol solution, as shown in Figure 2. The cell containing a polyDEAA gel particle was placed in a phenol solution in a 
Petri dish at $313 \mathrm{~K}$ until it reached a state of equilibrium. When the cell was quickly transferred to the other phenol solution set at $293 \mathrm{~K}$, the polyDEAA gel was exposed to the other phenol solution. That is, only temperature was varied, and the amount of adsorbed phenol was fixed. The other parts of the experimental procedure were carried out as described previously in Section 2.2.

Figure 2. Special design of observation cell for temperature swing with simultaneous change of phenol solution.

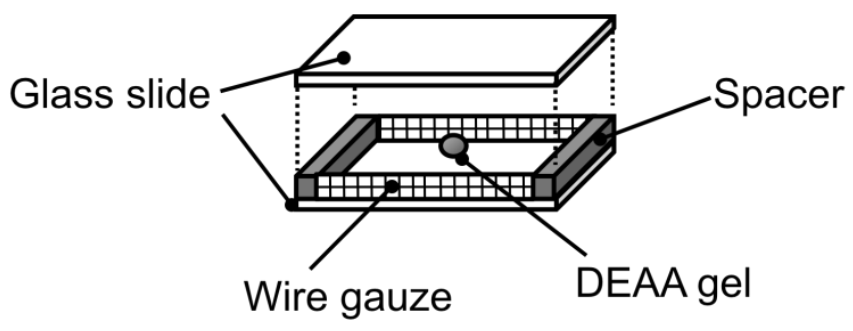

\section{Results and Discussion}

\subsection{Equilibrium Radius and Swelling Behavior of DEAA Gel in Phenol Solution}

The equilibrium radius of the DEAA gel particle was measured at various bulk phenol concentrations. Figure 3 shows the equilibrium radius change with bulk phenol concentration. The equilibrium radius $a_{\mathrm{e}}$ of this DEAA gel particle in pure water $\left(0 \mathrm{~g} / \mathrm{m}^{3}\right.$ phenol $)$ was $0.417 \mathrm{~mm}$ at $293 \mathrm{~K}$. The $a_{\mathrm{e}}$ value decreased slightly with increasing bulk phenol concentration, and was $0.381 \mathrm{~mm}$ at a bulk phenol concentration of $802 \mathrm{~g} / \mathrm{m}^{3}$. Between 874 and $916 \mathrm{~g} / \mathrm{m}^{3}$, the $a_{\mathrm{e}}$ value was discontinuously changed. The $a_{\mathrm{e}}$ was less than $0.3 \mathrm{~mm}$ at phenol concentrations above $950 \mathrm{~g} / \mathrm{m}^{3}$. A discontinuous change in characteristic length of DEAA gel at a certain phenol concentration has also been observed at a different temperature in the literature [25]. The temperature induced volume phase transition of hydrogels depends on the solute concentration (NIPA-phenol system) [26] and that the phenol concentration required to induce the volume phase transition for DEAA gel is lower than that for NIPA gel [25]. Our results imply that the phenol concentration required to induce a volume phase transition at $293 \mathrm{~K}$ is about $874 \mathrm{~g} / \mathrm{m}^{3}$ for the DEAA gel used in this study. At $313 \mathrm{~K}$, changing the phenol concentration only causes a small change in $a_{\mathrm{e}}$. The DEAA gel remains in the shrunken state at $313 \mathrm{~K}$. The reduction of the $a_{\mathrm{e}}$ value with increasing the bulk phenol concentration is attributed to the increase in hydrophobic interactions caused by the adsorption of phenol molecules onto the DEAA gel network. High hydrophobic interaction of DEAA and phenol promotes the hydrogen bond formation between amide group of gel and the hydroxyl group of phenol, and subsequent the adsorption of phenol divests the amide group of hydrated water molecules [20,24]. We now know that hydrophobic pollution is capable of reducing hydrogel size in a chemical sensor. Furthermore, temperature sensitivity of DEAA gel is also changed because the phenol adsorption to DEAA gel lowers the transition temperature [20,25]. 
Figure 3. Effect of phenol concentration on DEAA gel radius.

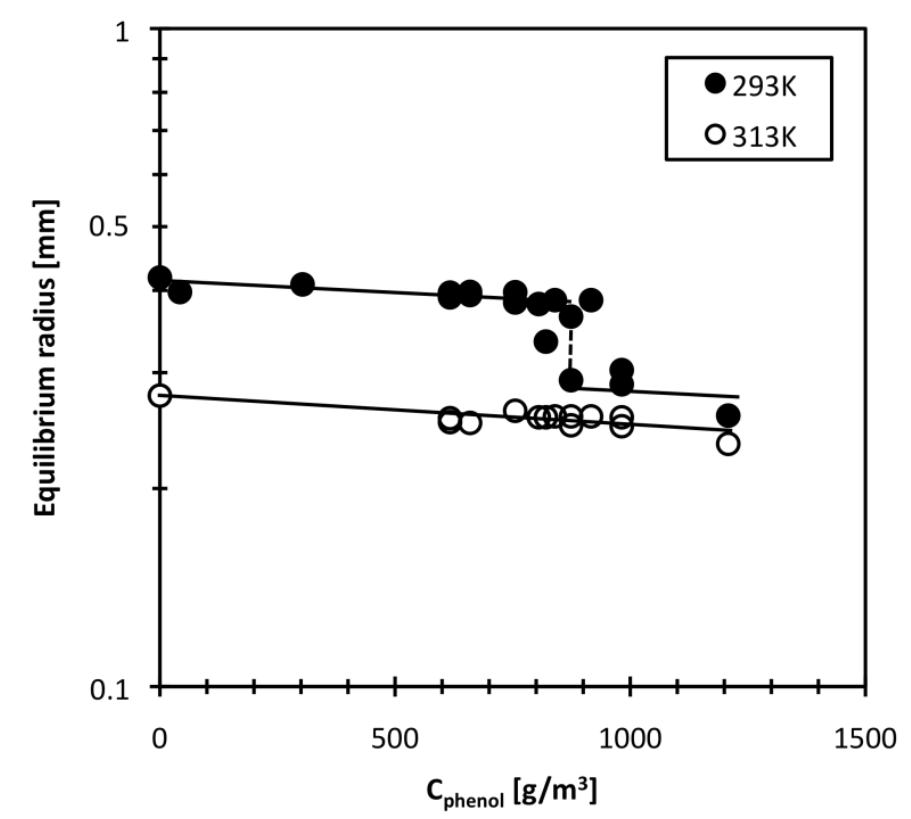

Figure 4 shows typical kinetic curves for the DEAA gel in phenol solution during the swelling process at $293 \mathrm{~K}$. In phenol solution, the kinetic curve showed three distinct stages with different slopes. The entire kinetic curve in phenol solution, therefore, could not be fitted with Equation (1), although the kinetic curve data below $306 \mathrm{~K}$ corresponded approximately to the theoretical curve for swelling in water [21]. A linear approximation could be fitted to the later stages of the curve, 1-2 $\mathrm{h}$ after the start of swelling process (stage (C) in Figure 4). Both the phenol adsorption onto DEAA gel and desorption from the gel processes were almost finished within $2 \mathrm{~h}$ (data not shown). This time, range is very similar to the time of the change from stages (B) to (C) shown in Figure 4. The lack of fitting during the earlier stages suggests attributions of phenol adsorption-desorption process and subsequent polymer chain network relaxation during stages (A) and/or (B). The effect of hydrophobic pollution on hydrogel behavior changes with time.

Figure 4. Typical kinetic curve of DEAA gel during swelling in phenol solution. Conditions: bulk phenol concentrations $755 \mathrm{~g} / \mathrm{m}^{3}$; temperature swing from $313 \mathrm{~K}$ to $293 \mathrm{~K}$.

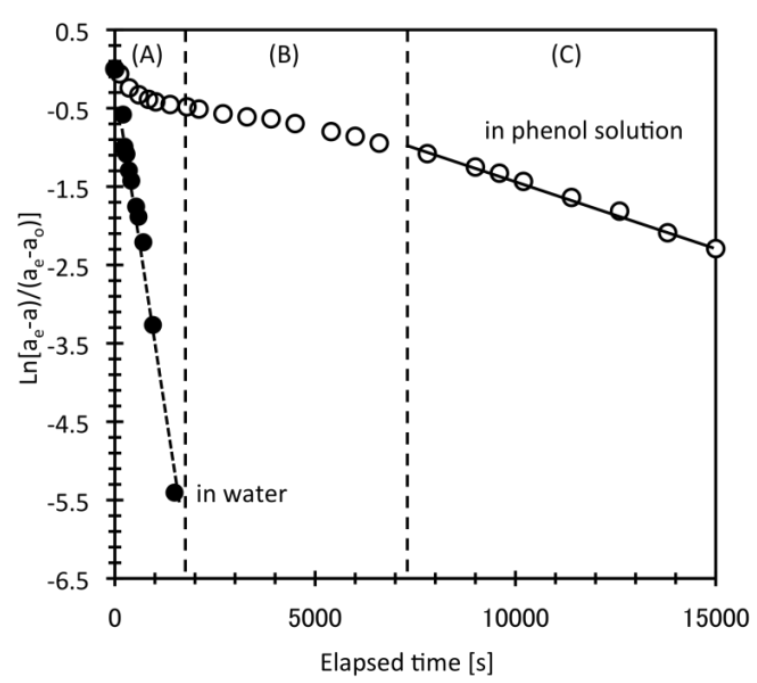




\subsection{Effect of Phenol Adsorption-Desorption Process on Swelling Behavior}

If the temperature swings from $313 \mathrm{~K}$ to $293 \mathrm{~K}$ under an excess phenol concentration (that is, when the bulk phenol concentration is constant as indicated by arrow (a) in Figure 1), the amount of adsorbed phenol on the DEAA gel is reduced according to the adsorption isotherm. To elucidate the effect of the phenol adsorption-desorption process on swelling behavior, the temperature swing experiment was performed under conditions that the amount of adsorbed phenol is fixed. As indicated by arrow (b) in Figure 1, the bulk phenol concentration was simultaneously changed with temperature swing to keep the amount of adsorbed phenol constant, at $q_{\text {phenol }}=42 \mathrm{~g} / \mathrm{kg}$ of dry gel. The phenol concentration $C_{\text {phenol }}$ was changed from $520 \mathrm{~g} / \mathrm{m}^{3}$ at $313 \mathrm{~K}$ to $850 \mathrm{~g} / \mathrm{m}^{3}$ at $293 \mathrm{~K}$.

Figure 5 shows the radius change of a DEAA gel particle and kinetic curve for temperature swing from $313 \mathrm{~K}$ to $293 \mathrm{~K}$ under fixed $q_{\text {phenol }}$ condition. The data obtained with fixed bulk phenol concentration $\left(C_{\text {phenol }}=850 \mathrm{~g} / \mathrm{m}^{3}\right)$ were also plotted for comparison. The final DEAA gel radius under fixed $q_{\text {phenol }}$ condition finally reached same length as the radius obtained at phenol concentration of $850 \mathrm{~g} / \mathrm{m}^{3}$ because the final amount of adsorbed phenol was the same for both fixed $q_{\text {phenol }}$ and fixed $C_{\text {phenol }}$ conditions (Figure 5a). The initial amount of adsorbed phenol under fixed $q_{\text {phenol }}$ condition is lower than that under fixed $C_{\text {phenol }}$ conditions as shown in Figure 1. During the initial stages, therefore, the DEAA gel radius under fixed $q_{\text {phenol }}$ condition was rapidly varied. The kinetic curve under fixed $q_{\text {phenol }}$ condition was a typical curve that could be described by the Tanaka and Fillmore theory $[17,18]$, and consequently the linear approximation based on Equation (1) was successful (Figure 5b). This result implies that phenol was not eliminated from the DEAA gel and the polymer network decorated with fixed amount of adsorbed phenol apparently diffused. The slope of the approximately linear region obtained under fixed $q_{\text {phenol }}$ condition was very similar to the slope of the curve obtained under fixed $C_{\text {phenol }}$ condition. This result is evidence that the polymer network decorated with fixed amount of phenol is undergoing collective diffusion in the later stage (C) of the curves obtained under fixed bulk phenol concentration and that the phenol adsorption-desorption process has finished before the start of stage (C). Phenol adsorption-desorption proceeds during the earlier stages of swelling, while the DEAA gel continues to swell after the phenol adsorption-desorption has reached a state of equilibrium. This is attributed to the slower kinetics of polymer network diffusion and relaxation compared with those of phenol adsorption.

The above results in phenol solution can be explained as follows. In the first stage (A), the volume change in the DEAA gel is extreme as a result of the sudden change in surrounding temperature. In the second stage (B), the volume change is suppressed by the adsorption-desorption of phenol molecules. After the phenol adsorption-desorption process and polymer network relaxation are almost complete, the DEAA polymer network decorated with phenol molecules [27,28] swells during the last stage (C). The apparent collective polymer diffusion constant obtained from the data in stage (C), therefore, can be regarded as the collective diffusion constant of the DEAA polymer network decorated with phenol molecules. 
Figure 5. Comparison of kinetic curve for DEAA gel swelling process under two different types of conditions: fixed $q_{\text {phenol }}$ or fixed $C_{\text {phenol }}$. (a) Time-course of DEAA gel radius (b) Kinetic curve during swelling process. Conditions: bulk phenol concentration $850 \mathrm{~g} / \mathrm{m}^{3}$; temperature swing from $313 \mathrm{~K}$ to $293 \mathrm{~K}$.

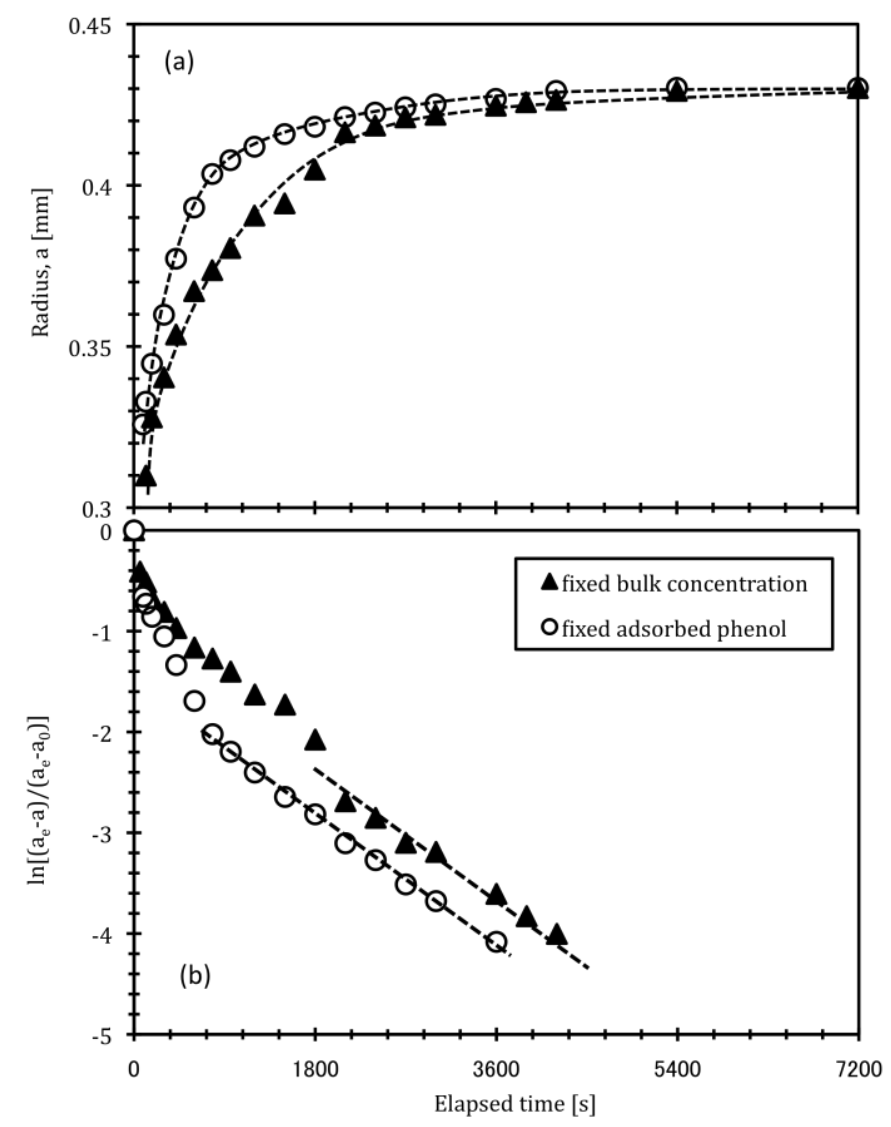

\subsection{Effect of Phenol Adsorption on Collective Polymer Diffusion Constant}

By fitting the data in stage (C) to Equation (1), the apparent collective polymer chain diffusion constant was obtained. Figure 6 shows the ratio of collective polymer diffusion constant in phenol solution, $D$, to that in pure water, $D_{0}$, as a function of bulk phenol concentration $C_{\text {phenol }}$. As shown and discussed in earlier sections, the phenol concentration changed the $a_{\mathrm{e}}$ value of the DEAA gel discontinuously at the transition concentration $C_{\mathrm{tr}}$, about $874 \mathrm{~g} / \mathrm{m}^{3}$. The $D$ value decreased strongly with increase in bulk phenol concentration up to $C_{\mathrm{tr}}$ (open circles). When the phenol concentration was higher than $C_{\text {tr }}$, the $D$ value increased (closed circles). This behavior is quite similar to the "critical slowing down" of the $D$ value with swelling temperature found for NIPA gel $[19,29]$. The volume phase transition of DEAA gel is continuous with temperature [21,25], but the volume changes discontinuously with phenol adsorption. Although the swing temperature is fixed, the amount of adsorbed phenol varies with phenol concentration, as shown in Figure 1. The behavior of the $D$ value will be affected by the amount of adsorbed phenol and the phenol distribution in the DEAA gel particle. 
Figure 6. Relationship between collective diffusion constant and bulk phenol concentration.

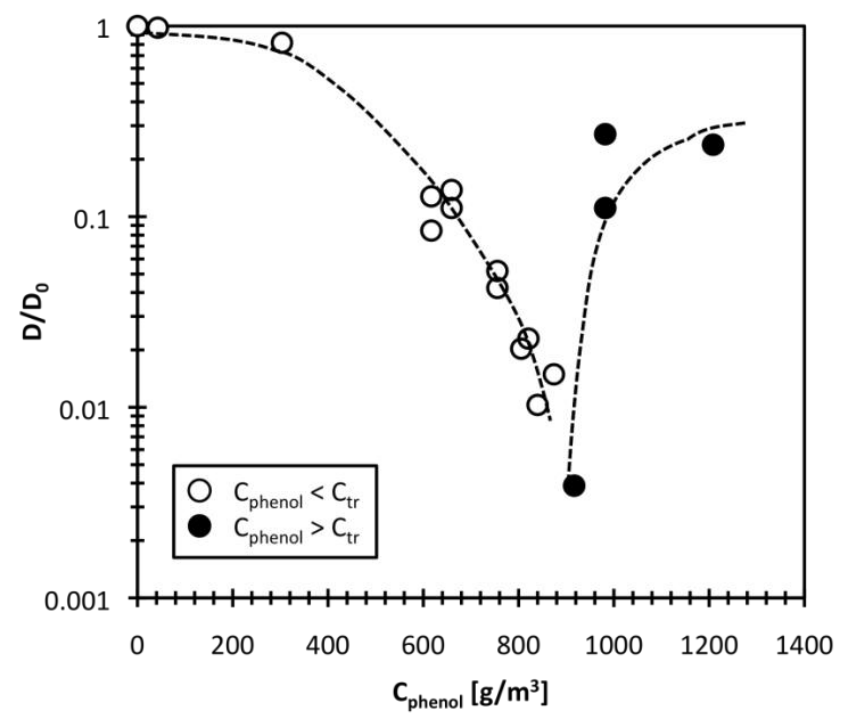

When considering the temperature dependence of the collective polymer diffusion constant, we can use the reduced temperature, $E[29,30]$ :

$$
E=\frac{\left|T-T_{t r}\right|}{T_{t r}}
$$

where $T_{\text {tr }}$ is the transition temperature. The $D$ value is related to $E$ by Equation (5):

$$
D \propto E^{v}
$$

where $v$ is a fitted exponent. This equation is shown to be associated with the mesh size of polymer networks, friction coefficient $f$, and solution viscosity $\eta$ [29]. Here, an analogous relation is defined using the bulk phenol concentration instead of temperature. The phenol concentration difference $E_{C}$ is analogous to the reduced temperature, $E$ :

$$
E_{C}=\frac{\left|C_{\text {phenol }}-C_{t r}\right|}{C_{t r}}
$$

The phenol adsorption amount $q_{\text {phenol }}$ depends on the phenol concentration $C_{\text {phenol }}$ from Equation (1).

$$
C_{\text {phenol }} \propto q_{\text {pheno }}^{N}
$$

Therefore, the phenol concentration difference $E_{C}$ is also a function of $q_{\text {phenol. Assuming that }}$ $C_{\text {tr }}=874 \mathrm{~g} / \mathrm{m}^{3}$, the diffusion constant ratio $D / D_{0}$ was $\log -\log$ plotted against $E_{C}$ in Figure 7. In principle, the $D$ value may have to be normalized with the solution viscosity $\eta$ to be able to examine the influence of mesh size, as shown in previous research [21]. In this study, the solution viscosity change with phenol concentration is negligible because the solution viscosity at low phenol concentrations $(\eta)$ can be approximated as the water viscosity $\left(\eta_{0}\right)$ [27]. Therefore, the diffusion constant ratio $D / D_{0}$ is equal to $D \eta / D_{0} \eta_{0}$ and the effect of solution viscosity is eliminated. As shown in Figure 7 , the $D$ value depends strongly on $E_{C}$, especially when $C_{\text {phenol }}<C_{\text {tr }}$ (open circles). When $C_{\text {phenol }}>C_{\text {tr }}$, the $D$ data may be scattered (closed circles) because the gel size change is very small and complicated. From the fitted curves, $v=1.48$ for swelling. The collective polymer diffusion constant in 
phenol solution is correlated well with the phenol concentration difference based on the transition concentration, which indicates that the diffusion constant is mainly affected in phenol solution by the amount of adsorbed phenol and the mesh size of the polymer networks.

Figure 7. Effect of the reduced concentration $E_{C}$ on the collective diffusion constant.

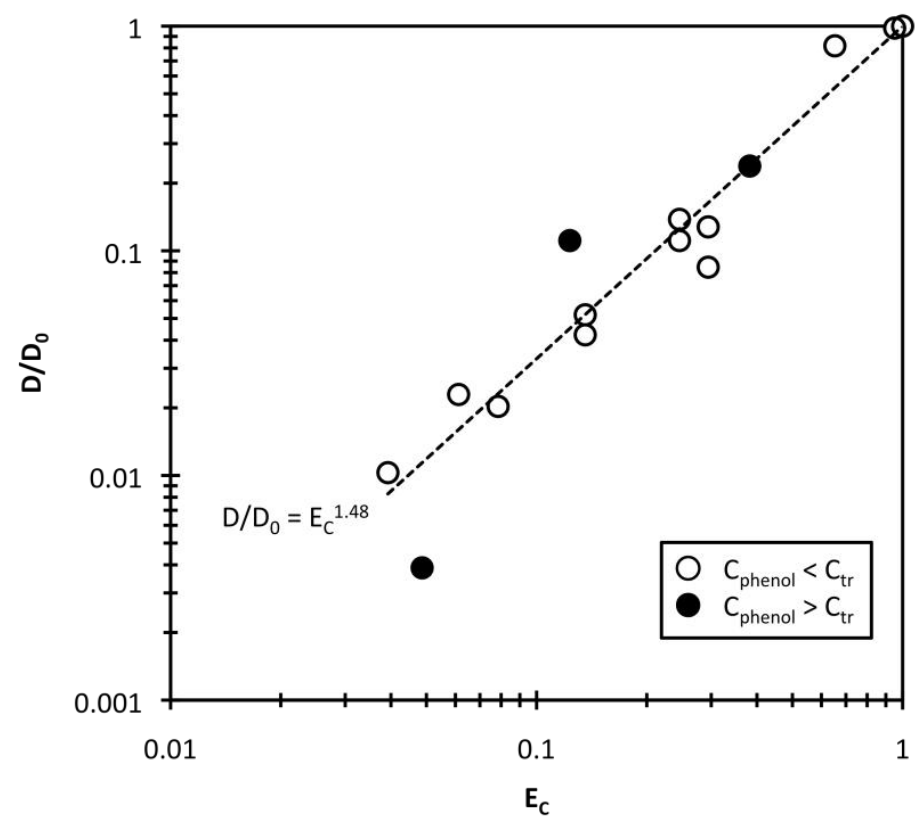

\section{Conclusions}

We investigated the effect of hydrophobic pollution on hydrogel response in aqueous solution with use of $\mathrm{N}, \mathrm{N}$-diethylacrylamide gel as a hydrogel and phenol as a hydrophobic pollution. The equilibrium radius of DEAA gel in dilute phenol solution changed discontinuously at phenol concentration of about $874 \mathrm{~g} / \mathrm{m}^{3}$. The collective polymer diffusion constant was dramatically decreased in the range of 874-916 g/m $\mathrm{m}^{3}$, suggesting a "critical slowing down". When comparing kinetic curves, we find that the slope of the approximately linear region obtained under conditions of fixed $q_{\text {phenol }}$ is very similar to the slope of the curve obtained under conditions of fixed $C_{\text {phenol }}$. During the latter stages of swelling, we found that a polymer network decorated with a constant amount of phenol exhibits collective diffusion. Phenol adsorption-desorption proceeds during the earlier stages of swelling, while the DEAA gel continues to swell after the phenol adsorption-desorption finished. Using the phenol concentration difference $E_{C}$ (analogous to the reduced temperature, $E$ ) we find that the $D$ value depends strongly on $E_{C}, D \propto E_{C}^{v}$, and that the exponent $v=1.48$. The correlation will be useful as estimation of hydrogel response reduction associated with hydrophobic pollution.

\section{Acknowledgments}

The authors are grateful to the Sasaki Environment Tech. Foundation, Japan, for supporting a part of this research. 


\section{Conflicts of Interest}

The authors declare no conflict of interest.

\section{References}

1. Shin, J.; Braun, P.V.; Lee, W. Fast response photonic crystal $\mathrm{pH}$ sensor based on templated photo-polymerized hydrogel inverse opal. Sens. Actuators B 2010, 150, 183-190.

2. Lee, S.; Ibey, B.L.; Coté, G.L.; Pishko, M.V. Measurement of pH and dissolved oxygen within cell culture media using a hydrogel microarray sensor. Sens. Actuators B 2008, 128, 388-398.

3. Pi, S.; Ju, X.; Wu, H.; Xie, R.; Chu, L. Smart responsive microcapsules capable of recognizing heavy metal ions. J. Colloid Interface Sci. 2010, 349, 512-518.

4. Herber, S.; Olthuis, W.; Bergveld, P. A swelling hydrogel-based $\mathrm{P}_{\mathrm{CO} 2}$ sensor. Sens. Actuators B 2003, 91, 378-382.

5. Meskath, S.; Urban, G.; Heinzea, J. A new optochemical chlorine gas sensor based on the application of amphiphilic co-networks as matrices. Sens. Actuators B 2011, 151, 327-332.

6. Zguris, J.; Pishko, M.V. Nitric oxide sensitive fluorescent poly(ethylene glycol) hydrogel microstructures. Sens. Actuators B 2006, 115, 503-509.

7. Endo, T.; Yanagida, Y.; Hatsuzawa, T. Colorimetric detection of volatile organic compounds using a colloidal crystal-based chemical sensor for environmental applications. Sens. Actuators $B$ 2007, 125, 589-595.

8. Yamagiwa, K.; Komi, T.; Kumakura, A.; Yokoyama, S.; Yoshida, M.; Ohkawa, A. Photo-crosslinked copolymer gel as an adsorbent for temperature-swing adsorption process. J. Chem. Eng. Jpn. 2004, 37, 1274-1278.

9. Yamagiwa, K.; Komi, T.; Yoshida, M.; Ohkawa, A.; Iida, T. Temperature-swing adsorption of nonionic surfactant with photo-crosslinked polymeric gel. J. Chem. Eng. Jpn. 2001, 34, 1171-1176.

10. Tokuyama, H.; Yanagawa, K.; Sakohara, S. Temperature swing adsorption of heavy metals on novel phosphate-type adsorbents using thermosensitive gels and/or polymers. Sep. Purif. Technol. 2006, 50, 8-14.

11. Tokuyama, H.; Kanehara, A. Temperature swing adsorption of gold(III) ions on poly(Nisopropylacrylamide) gel. React. Funct. Polym. 2007, 67, 136-143.

12. Takeshita, K.; Matsumura, T.; Nakao, Y. Separation of americium(III) and europium(III) by thermal-swing extraction using thermosensitive polymer gel. Prog. Nucl. Energy 2008, 50, 466-469.

13. Huber, D.L.; Manginell, R.P.; Samara, M.A.; Kim, B.; Bunker, B.C. Programmed adsorption and release of proteins in a microfluidic device. Science 2003, 301, 352-354.

14. Klouda, L.; Mikos, A.G. Thermoresponsive hydrogels in biomedical applications. Eur. J. Pharm. Biopharm. 2008, 68, 34-45.

15. Sershen, S.; West, J. Implantable, polymeric systems for modulated drug delivery. Adv. Drug Deliv. Rev. 2002, 54, 1225-1235. 
16. Tokuyama, H.; Kanazawa, R.; Sakohara, S. Equilibrium and kinetics for temperature swing adsorption of a target metal on molecular imprinted thermosensitive gel adsorbents. Sep. Purif. Technol. 2005, 44, 152-159.

17. Tanaka, T.; Fillmore, J. Kinetics of swelling of gels. J. Chem. Phys. 1979, 70, 1214-1218.

18. Li, Y.; Tanaka, T. Kinetics of swelling and shrinking of gels. J. Chem. Phys. 1990, 92, 1365-1371.

19. Sato Matsuo, E.; Tanaka, T. Kinetics of discontinuous volume-phase transition of gels. J. Chem. Phys. 1988, 89, 1695-1703.

20. Kosik, K.; Wilk, E.; Geissler, E.; Laszlo, K. Interaction of phenols with thermo-responsive hydrogels. Colloids Surf. A Physicochem. Eng. Aspects 2008, 319, 159-164.

21. Tajima, H.; Yoshida, Y.; Yamagiwa, K. Experimental study of swelling and shrinking kinetics of spherical poly (N,N-diethylacrylamide) gel with continuous phase transition. Polymer 2011, 52, 732-738.

22. Tajima, H.; Yoshida, Y.; Abiko, S.; Yamagiwa, K. Size adjustment of spherical temperature-sensitive hydrogel beads by liquid-liquid dispersion using a Kenics static mixer. Chem. Eng. J. 2010, 156, 479-486.

23. Idziak, I.; Avoce, D.; Lessaed, D.; Grabel, D.; Zhu, X.X. Thermosensitivity of aqueous solutions of poly(N,N-diethylacrylamide). Macromolecules 1999, 32, 1260-1263.

24. Kawashima, T.; Koga, S.; Annaka, M.; Sasaki, S. Roles of hydrophobic interaction in a volume transition of alkylacrylamide gel induced by the hydrogen-bond-driving alkylphenol binding. J. Phys. Chem. B 2005, 109, 1055-1062.

25. Koga, S.; Kawashima, T.; Sasaki, S. Elastic relaxation of collapsed poly(alkylacrylamide) gels and their complexes with phenol. J. Phys. Chem. B 2004, 108, 10838-10844.

26. Suzuki, Y.; Suzuki, N.; Takasu, Y.; Nishio, I. A study on the structure of water in an aqueous solution by the solvent effect on a volume phase transition of $\mathrm{N}$-isopropylacrylamide gel and low-frequency Raman spectropy. J. Chem. Phys. 1997, 107, 5890-5897.

27. Laszio, K.; Kosik, K.; Rochas, C.; Geissler, E. Phase transition in Poly (N-isopropylacrylamide) hydrogels induced by phenols. Macromolecules 2003, 36, 7771-7776.

28. Kosik, K.; Geissler, E.; Zrinyi, M.; Laszlo, K. Interaction of non-ionic hydrogels with small aromatic molecules. Polym. Adv. Technol. 2003, 14, 771-775.

29. Takahashi, K.; Takigawa, T.; Masuda, T. Swelling and deswelling kinetics of poly(Nisopropylacrylamide) gels. J. Chem. Phys. 2004, 120, 2972-2979.

30. Tokita, M.; Tanaka, T. Reversible decrease of gel-solvemt friction. Science 1991, 253, 1121-1123.

(C) 2013 by the authors; licensee MDPI, Basel, Switzerland. This article is an open access article distributed under the terms and conditions of the Creative Commons Attribution license (http://creativecommons.org/licenses/by/3.0/). 